\title{
Preventive effects of garlic (Allium sativum) on oxidative stress and histopathology of cardiac tissue in streptozotocin-induced diabetic rats
}

\author{
R Naderi ${ }^{1}$, G Mohaddes ${ }^{1}$, M Mohammadi ${ }^{1}$, A Alihemmati ${ }^{2}$, R Badalzadeh ${ }^{1}$, \\ R Ghaznavi $^{3}$, R Ghyasi ${ }^{1}$, Sh Mohammadi ${ }^{4}$ \\ ${ }^{1}$ Drug Applied Research Center of Tabriz University of Medical Sciences, Tabriz, Iran \\ ${ }^{2}$ Department of Histology and Embryology, Faculty of Medicine, Tabriz University of Medical Sciences, \\ Tabriz, Iran \\ ${ }^{3}$ Department of Physiology, School of Medicine, Tehran University of Medical Sciences, Tehran, Iran \\ ${ }^{4}$ Student of pharmacy, Faculty of Pharmacy, Tabriz University of Medical Sciences, Tabriz, Iran
}

Received: September 27, 2014

Accepted: September 5, 2015

\begin{abstract}
Since some complications of diabetes mellitus may be caused or exacerbated by an oxidative stress, the protective effects of garlic (Allium sativum) were investigated in the blood and heart of streptozotocin-induced diabetic rats. Twenty-eight male Wistar rats were randomly divided into four groups: control, garlic, diabetic, and diabetic + garlic. Diabetes was induced by intraperitoneal (i.p.) injection of streptozotocin $(50 \mathrm{mg} / \mathrm{kg})$ in male rats. Rats were fed with raw fresh garlic homogenate $(250 \mathrm{mg} / \mathrm{kg})$ six days a week by gavage for a period of 6 weeks. At the end of the 6 th week blood samples and heart tissues were collected and used for determination of glutathione peroxidase (GPx), superoxide dismutase (SOD), catalase (CAT), malondialdehyde (MDA) and histological evaluation. Induction of diabetes increased MDA levels in blood and homogenates of heart. In diabetic rats treated with garlic, MDA levels decreased in blood and heart homogenates. Treatment of diabetic rats with garlic increased SOD, GPX and CAT in blood and heart homogenates. Histopathological finding of the myocardial tissue confirmed a protective role for garlic in diabetic rats. Thus, the present study reveals that garlic may effectively modulate antioxidants status in the blood and heart of streptozotocin induced-diabetic rats.
\end{abstract}

Keywords: diabetes, garlic, oxidative stress, heart, blood, rat

Diabetes mellitus is a metabolic disorder characterized by chronic hyperglycemia with impaired metabolism of carbohydrate, fat, and protein resulting from defects in insulin secretion or insulin action or both (13). Hyperglycemia exacerbates oxidative stress which is linked to diabetic complications (5). Cells have enzymatic and non-enzymatic scavenger systems against free radicals. Nevertheless, if free radical production and scavenger systems somehow become unbalanced, cells are exposed to oxidative damage resulting in cell injury (11).

Hyperglycemia as the major contributing factor of increased reactive oxygen species (ROS) production causes tissue damage or diabetic complications. Hyperglycemia activates protein kinase $\mathrm{C}$ (PKC) and Poly-ADP ribose polymerase (PARP) pathways; it also enhances formation of advanced glycation end products (AGEs) and polyol and hexosamine pathways. These pathways are activated or enhanced due to mitochondrial superoxide overproduction (12).

Corresponding author: Dr. Mustafa Mohammadi

Drug Applied Research Center of Tabriz University of Medical Sciences, Tabriz, Iran

Phone/Fax:+984113364664; E-mail: m.mohammadin@yahoo.com 
These mechanisms, which are sources of free radicals of oxygen, are taken into consideration when trying to explain the pathogenesis of many of the complications in diabetes, especially those related to the vascular endothelium, retina, peripheral nervous system, and cardiovascular system $(5,13)$.

Dietary plants, which have protective biochemical function of natural antioxidants, are suitable for prevention or protection against oxidative damage caused by free radical species (17). Among the known biological antioxidants, garlic (Allium sativum) has been used in herbal medicine for the treatment or prevention of a number of diseases such as cancer, cardiovascular disease and diabetes (17). It has been found to provide alleviating effects on hyperglycemia and hyperlipidemia. Garlic was also discovered to enhance the antioxidant status by lowering the level of reactive oxygen species and stimulating the production of antioxidant enzymes (7).

The present study was designed to investigate the effect of garlic treatment on oxidative stress and antioxidant balance in the blood and heart of streptozotocin-induced diabetic rats using both biochemical and histological parameters.

\section{Materials and Methods}

Animals and experimental design

Male Wistar rats (200-250 g) were housed four per cage and maintained on a $12 \mathrm{~h}$ light-dark cycle in an air conditioned constant temperature $\left(22 \pm 1^{\circ} \mathrm{C}\right)$ room. Food and water was made available ad libitum. The Ethic Committee for Animal Experiments of Tabriz University of Medical Sciences approved the study plan, and all experiments were conducted in accordance with the National Institute of Health Guide for the Care and Use of Laboratory Animals.

Twenty eight rats were randomly divided into four experimental groups $(n=7)$ :

1. Control,

2. Garlic: animals that received $250 \mathrm{mg} / \mathrm{kg}$ of homogenized garlic for 6 weeks,

3. Diabetic: animals that received $50 \mathrm{mg} / \mathrm{kg}$ of streptozotocin intraperitoneally (i.p.),

4. Diabetic + Garlic: animals that received single i.p. injection of $50 \mathrm{mg} / \mathrm{kg}$ of streptozotocin and $250 \mathrm{mg} / \mathrm{kg}$ of homogenized garlic for 6 weeks.

In order to induce diabetes, streptozotocin (STZ, Sigma-Aldrich, Steinheim, Germany) was dissolved in saline and a single i.p. dose $(50 \mathrm{mg} / \mathrm{kg})$ of STZ was injected to each animal. To confirm the induction of diabetes, 3 days after the STZ injection, blood glucose levels were determined from blood samples obtained by tail prick using a strip-operated glucometer (Elegance, CT-X10, Frankenberg, Germany). Animals with blood glucose levels higher than $300 \mathrm{mg} / \mathrm{dl}$ were selected. The control animals were injected with $0.4 \mathrm{~mL}$ of sodium citrate buffer, $\mathrm{pH} 4.5$.

Rats were fed with raw fresh garlic homogenate $(250 \mathrm{mg} / \mathrm{kg})$ by gavage 6 days a week for a period of 6 weeks. At the end of the 6 th week, all rats were deeply anesthetized with pentobarbital sodium (Sigma-Aldrich, Steinheim, Germany). Thirty-five mg/kg, i.p., then blood samples and heart tissues were collected from fasted animals before the rats were sacrificed.

Preparing garlic homogenate

Garlic (Allium sativum) bulbs were purchased from a local market. Cloves were peeled, sliced, ground into a paste and then suspended in distilled water. The garlic homogenate was prepared freshly each day. 
Tissue processing and homogenate preparation

Blood samples were collected from the inferior vena cava and stored in tubes at $-70{ }^{\circ} \mathrm{C}$ for determination of blood SOD, GPX and CAT activities. Hearts were excised, frozen in liquid nitrogen and stored at deep freeze $\left(-70{ }^{\circ} \mathrm{C}\right)$ for later measurements. For antioxidant activity measurement heart samples were homogenized in $1.15 \% \mathrm{KCl}$ solution (Sigma-Aldrich, Steinheim, Germany). The homogenates were centrifuged at $1000 \mathrm{rpm}$ for $1 \mathrm{~min}$ at $4{ }^{\circ} \mathrm{C}$. The tissue homogenate was then stored at $-20{ }^{\circ} \mathrm{C}$ for glutathione peroxidase (GPx), superoxide dismutase (SOD) and catalase (CAT) activities and malondialdehyde (MDA) measurements $(19,25)$.

\section{Determination of antioxidant enzymes}

Whole blood samples were used for determination of GPX and SOD and CAT. Superoxide dismutase (SOD) activity was determined using a commercial kit (RANSOD, Randox co., Antrim., United Kingdom) according to Delmas-Beauvieux et al. SOD activity was measured at $505 \mathrm{~nm}$ by a spectrophotometer (Pharmacia Biotech., Cambridge, England). In this method, xanthine and xanthine oxidase were used to generate superoxide radicals that react with 2-(4-iodophenyl)-3 (4-nitrophenol)-5-phenyl tetrazolium chloride (ITN) to form a red formazan dye. Concentrations of substrates were $0.05 \mathrm{mmol} / \mathrm{L}$ for xanthine and $0.025 \mathrm{mmol} / \mathrm{L}$ for ITN. SOD activity was measured by the degree of inhibition of this reaction. After calculating the percent of inhibition by using the related formula, SOD activity value was calculated by comparing with the standard curve and was expressed as U/g hemoglobin $(\mathrm{Hb})$ in blood and $\mathrm{U} / \mathrm{mg}$ protein in tissue $(19,25)$.

Glutathione peroxidase (GPX) activity was determined using commercial kit (RANSEL, Randox co., Antrim, United Kingdom) according to the method of Paglia and Valentine. GPX catalyses the oxidation of glutathione (at a concentration of $4 \mathrm{mmol} / \mathrm{L}$ ) by cumene hydroperoxide. In the presence of glutathione reductase (at a concentration $\geq 0.5$ units/L) and $0.28 \mathrm{mmol} / \mathrm{L}$ of NADPH, oxidized glutathione is immediately converted to the reduced form with concomitant oxidation of NADPH to NADP ${ }^{+}$. The decrease in absorbance at $340 \mathrm{~nm}$ $\left(37^{\circ} \mathrm{C}\right)$ was measured using a spectrophotometer (Pharmacia Biotech., Cambridge, England), and then GPx concentration was calculated by the related formula $(19,25)$.

\section{Malondialdehyde and catalase assessment}

Malondialdehyde (MDA) as the end-product of lipid peroxidation was measured in the blood samples and tissue extracts according to the Esterbauer and Cheeseman method. MDA reacts with thiobarbituric acid (Sigma-Aldrich, Steinheim, Germany) and produces a pink pigment that has a maximum absorption at $532 \mathrm{~nm}(19,25)$.

Catalase activity was measured using the Aebi method. According to this method, measurement was performed based on the dissociation rate of $\mathrm{H}_{2} \mathrm{O}_{2}$ in $240 \mathrm{~nm}$ at $20{ }^{\circ} \mathrm{C}$. Myocardial homogenate aliquots and whole blood lysates were centrifuged. The adequate amount of supernatant was added to a reaction mixture that contained $0.002 \%$ Triton X-100, $0.1 \mathrm{mM}$ EDTA, $0.5 \mathrm{M}$ potassium phosphate buffer, and $15 \mathrm{mM} \mathrm{H}_{2} \mathrm{O}_{2}$ in $1 \mathrm{~mL}$ final volume at $\mathrm{pH}$ 7.0. Activity was calculated as the decomposition rate within the initial $15 \mathrm{~s}$ and expressed as $\mathrm{K} / \mathrm{gHb}$ in blood and $\mathrm{K} / \mathrm{mg}$ protein in heart (19).

\section{Histological evaluation}

Heart tissues were immediately isolated and fixed in $10 \%$ buffered-formalin solution, dehydrated in ascending grades of alcohol and embedded in paraffin. Sections of $5 \mu \mathrm{m}$ were 
taken, stained with hematoxylin-eosin (H-E), and examined under light microscope (Olympus BH-2, Tokyo, Japan) in a blinded manner. Heart tissues were evaluated in terms of interstitial edema and congestion, leukocytosis infiltration, and myonecrosis. Histological changes were scored on a 4-point scale: $(-)$ none, $(+)$ mild, $(++)$ moderate, and $(+++)$ severe damage (3).

\section{Statistical analysis}

Data were statistically analyzed using one-way analysis of variance (ANOVA) followed by Tukey's test. The significant level was set at $p<0.05$. Results are expressed as means $\pm \mathrm{SEM}$.

\section{Results}

Body weight and blood glucose

As shown in Table I, there was no significant difference in body weight among the four groups at the beginning of this research. Induction of diabetes by streptozotocin significantly $(p<0.001)$ decreased body weight in diabetic animals and the treatment with garlic for 6 weeks could not significantly increase it in diabetic+garlic group compared to the diabetic group.

Blood glucose levels increased significantly $(p<0.001)$ in the diabetic group compared to the control group. However, after 6 weeks of administration of garlic, blood glucose levels in the diabetic + garlic group was significantly $(p<0.001)$ lower than in the diabetic group.

Table I. Effects of diabetes and garlic on body weight and blood glucose concentrations

in streptozotocin-induced diabetic rats at first and after 6 weeks (means $\pm \mathrm{SEM}$ )

\begin{tabular}{|l|c|c|c|c|}
\hline Variants & Control & Garlic & Diabetic & Diabetic + Garlic \\
\hline Initial BW & & & & $236 \pm 6.1$ \\
\hline After 6 weeks BW & $230 \pm 5.6$ & $236 \pm 7.7$ & $241 \pm 3.9$ & $183 \pm 6.7$ \\
\hline Initial BG & $118 \pm 2.1$ & $128 \pm 1.3$ & $383 \pm 17.9^{* * *}$ & $381 \pm 24$ \\
\hline After 6 weeks BG & $115 \pm 1.6$ & $118 \pm 2.6$ & $434 \pm 19.8^{* * *}$ & $341 \pm 12.37^{\$ \$ \$}$ \\
\hline
\end{tabular}

$* * * p<0.001$ compared with control group. ${ }^{\$ \$} p<0.001$ compared with diabetic group.

BW: body weight (gr), BG: blood glucose (mg/dL)

\section{Lipid peroxidation}

A significant $(p<0.05)$ increase in MDA level was observed in heart and blood of the diabetic group in comparison with the control group. However, treatment of the diabetic group with garlic significantly decreased MDA levels in blood $(p<0.01)$ and heart homogenates $(p<0.05)$ (Fig. 1).

\section{Antioxidant enzymes}

There was no change in blood and heart SOD, GPx, and CAT activity in the diabetic group compared to the control group. The six-week administration of homogenized garlic $(250 \mathrm{mg} /$ $\mathrm{kg})$ caused significant increases in SOD $(p<0.01)$, GPX $(p<0.05)$, and CAT $(p<0.01)$ levels in blood and it also significantly elevated SOD $(p<0.01)$, GPX $(p<0.05)$, and CAT $(p<0.05)$ levels in heart homogentaes compared to the control group. After the same 
treatment in diabetic animals, antioxidant enzyme levels increased significantly in blood [SOD $(p<0.001), \operatorname{GPX}(p<0.05), \operatorname{CAT}(p<0.01)]$ and in the heart homogenates [SOD $(p<$ $0.01)$, GPX $(p<0.001)$, CAT $(p<0.01)]$ compared to the diabetic group. (Fig. 2a, b and c).
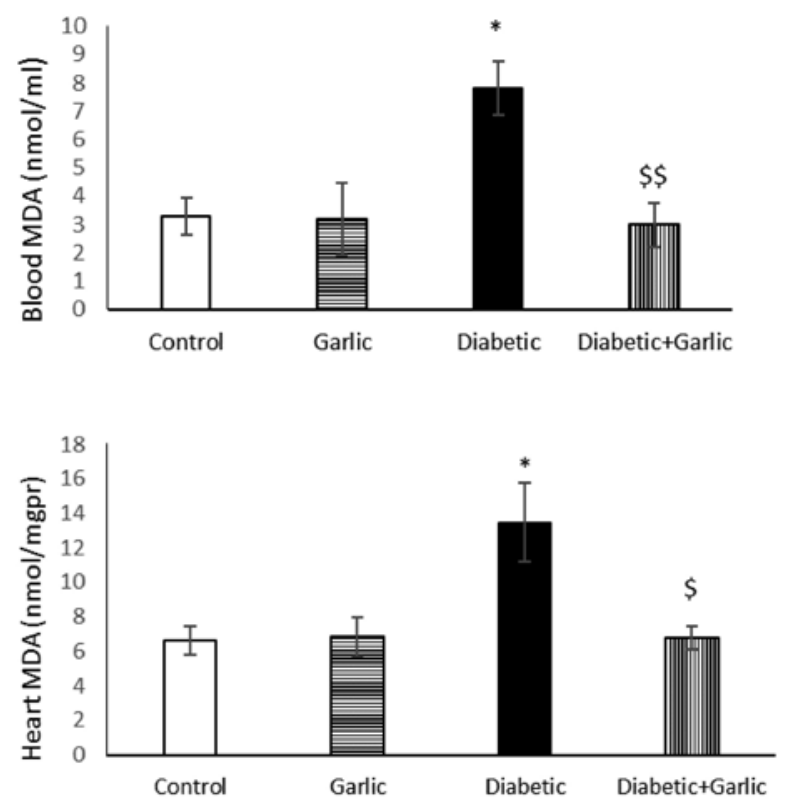

Fig. 1. Effects of diabetes and garlic treatment on MDA levels in blood samples and heart tissue of different groups. The values represent means $\pm \operatorname{SEM}(n=7)$.

${ }^{*} p<0.05$ versus control group. ${ }^{\$} p<0.05,{ }^{\$ \$} p<0.01$ versus diabetic group

A
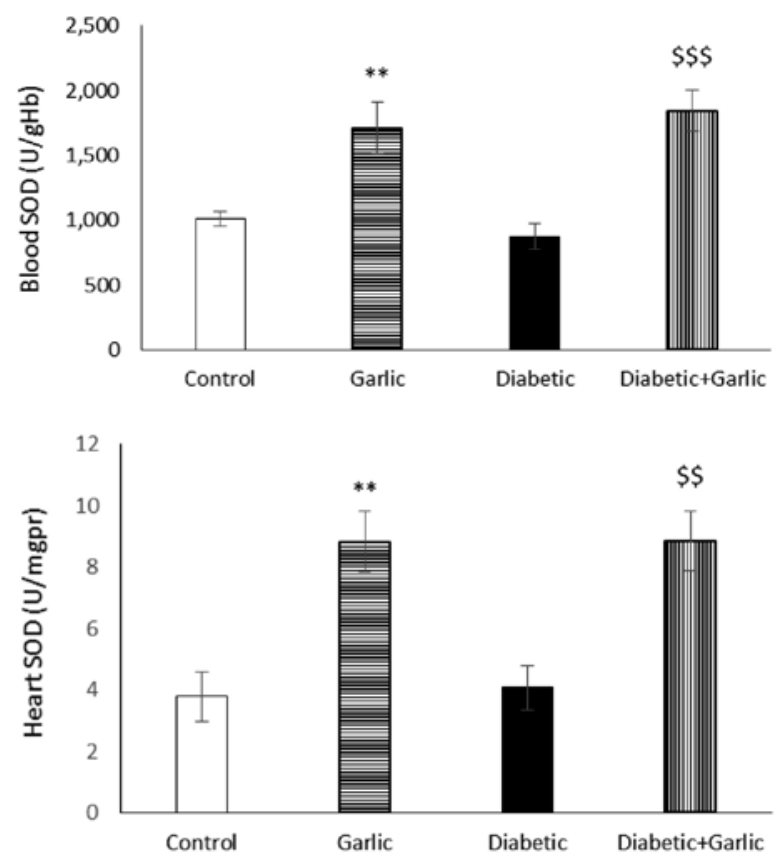
B
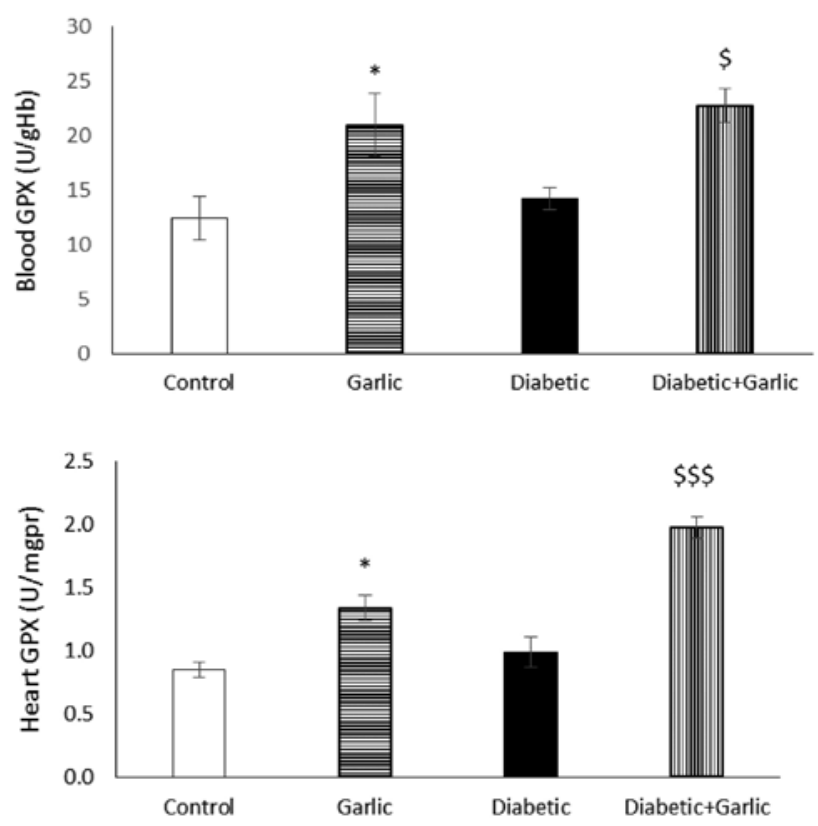

$\mathrm{C}$
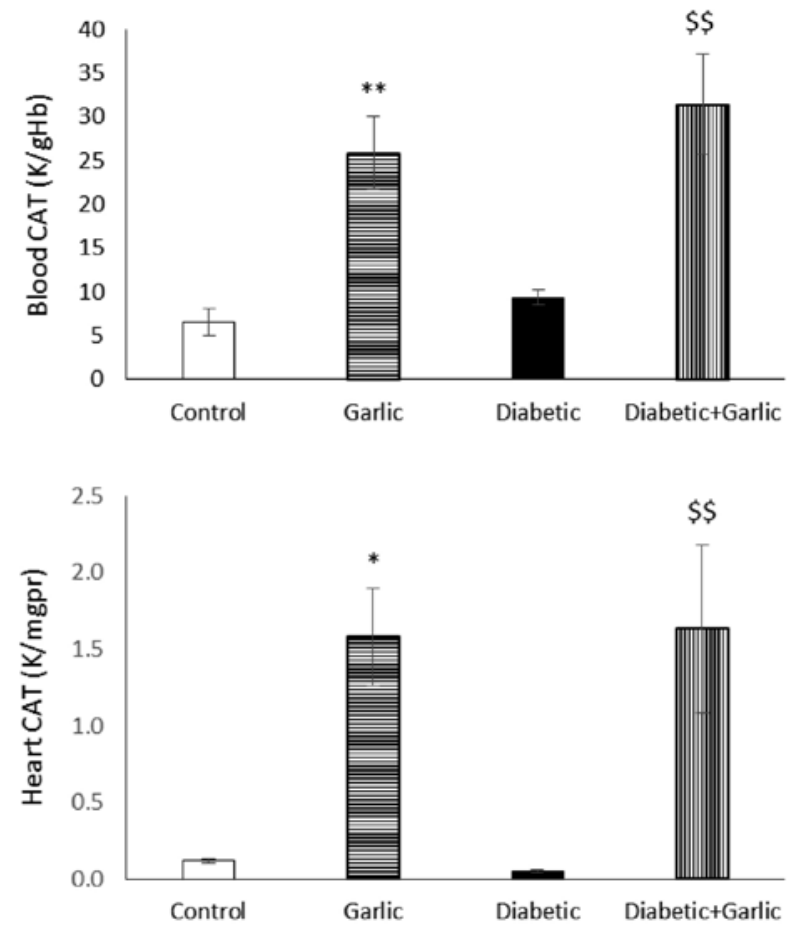

Fig. 2. Effect of diabetes and garlic treatment on antioxidant enzymes (a) SOD activity, (b) GPx activity and, (c) catalase activity in blood samples and heart tissues of different groups. The values represent means $\pm \operatorname{SEM}(n=7) .{ }^{*} p<0.05,{ }^{* *} p<0.01$ versus control group. ${ }^{\$} p<0.05,{ }^{\$} p<0.01,{ }^{\$ \$} p<0.001$ versus diabetic group 


\section{Histopathological findings}

Histological examination of the hearts are shown in Fig. 3. Figure 3a shows a normal heart tissue in the control group. There was no histological changes in the garlic group (Fig. 3b). In the diabetic group, interstitial edema, leukocyte infiltration, and myonecrosis were higher than those of the control group (Fig. 3c). Garlic treatment resulted in a marked attenuation of interstitial edema, leukocyte infiltration, and myonecrosis in diabetic animals (Fig. 3d, Table II).
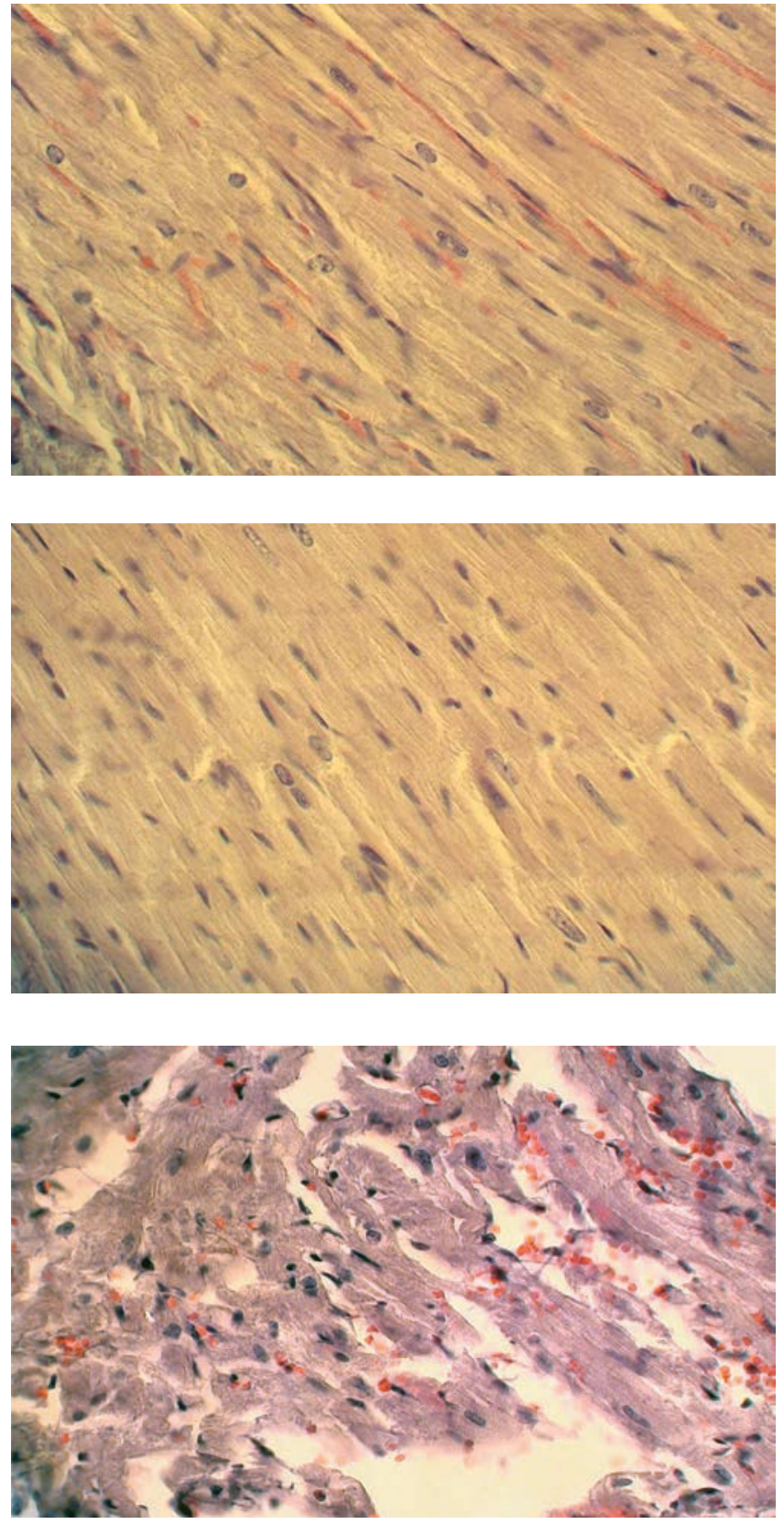

Fig. 3a. The normal heart tissue Histopathological evaluation of rat cardiomyocytes after 6 weeks.

Heart sections are stained by hematoxylin and eosin (HE) and examined by a light microscope. Healthy appearance of cardiomyocytes: normal morphology, normal cardiac fibers without any edema, and inflammatory cells $(40 \times \mathrm{HE})$

Fig. 3b. Heart tissue structure in the garlic group.

Histopathological evaluation of rat cardiomyocytes after 6 weeks.

Heart sections are stained by hematoxylin and eosin (HE) and examined by a light microscope.

Healthy appearance of cardiomyocytes: normal morphology, normal cardiac fibers without any edema, and inflammatory cells $(40 \times \mathrm{HE})$

\section{Fig. 3c. Heart tissue in} the diabetic group.

Histopathological evaluation of rat cardiomyocytes after 6 weeks. Heart sections are stained by hematoxylin and eosin (HE) and examined by a light microscope. Interstitial edema and congestion,

Leukocytosis infiltration, myonecrosis, separation and disorganization of cardiomyocytes $(40 \times \mathrm{HE})$ 


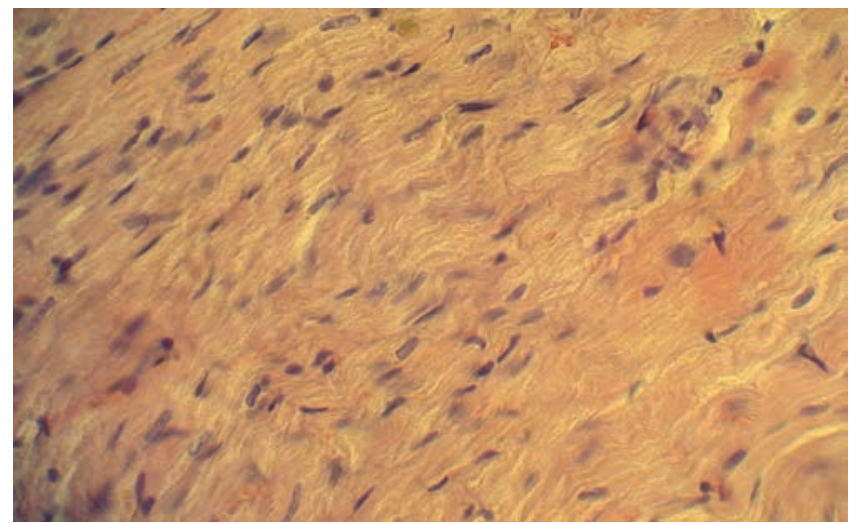

Fig. 3d. Heart tissue in the diabetic + garlic group.

Histopathological evaluation of rat cardiomyocytes after 6 weeks.

Heart sections are stained by hematoxylin and eosin (HE) and examined by a light microscope. Mild edema and necrosis with few inflammatory cells and separation of muscle fibers $(40 \times \mathrm{HE})$

Table II. Comparison of histological changes of cardiomyocytes among eight groups of rats under using of diabetes and/or garlic (Hematoxylin and Eosin)

\begin{tabular}{|l|c|c|c|}
\hline \multicolumn{1}{|c|}{ Groups } & $\begin{array}{c}\text { Interstitial edema and } \\
\text { congestion }\end{array}$ & Leukocyte infiltration & Cadiomyocyte necrosis \\
\hline Control & - & - & - \\
\hline Garlic & - & - & - \\
\hline Diabetic & +++ & +++ & +++ \\
\hline Diabetic+garlic & + & + & + \\
\hline
\end{tabular}

A minimum of 10 fields for each cardiomyocyte slide were examined and assigned for severity of changes using scores on a scale of: $(-)$ none, $(+)$ mild, $(++)$ moderate, and $(+++)$ severe damage $(n=7$ for each group)

\section{Discussion}

In this study, we found that streptozotocin injection in the diabetic rats led to a significant increase in plasma glucose compared to the control; and treatment of the diabetic rats with homogenized garlic modulated it. We also showed that homogenized garlic significantly decreased MDA level of heart and blood and also increased antioxidant enzymes (SOD, GPX and CAT) in the blood and heart homogenates of control and streptozotocin-induced diabetic rats suggesting that garlic has strong antioxidant effect in vivo. Also, histopathological findings showed that garlic treatment for 6 weeks decreased pathological changes in the heart of diabetic animals.

These findings are in agreement with the results of previous studies which reported that injection of aqueous garlic extract $(500 \mathrm{mg} / \mathrm{kg})$ increased total serum antioxidant levels (9) and incubation of methanol garlic extract $(500 \mathrm{mg} / \mathrm{kg})$ decreased hepatic MDA levels in STZ-treated diabetic rats (24).

Hyperglycemia of diabetes results in the formation of advanced glycation end products (AGEs) and production of reactive oxygen species (ROS) in different cell lines (20). Diabetes also induces oxidative stress by increased level of MDA and also diminished antioxidant enzymes activities including SOD, GPX, CAT $(4,26)$ that is suggested as a mechanism underlying diabetic complications (4). 
Since most of the antidiabetic medications could have side effects (8), many studies have been conducted to identify natural substances that show antidiabetic activity with less side effects $(22,27)$. Garlic and its products could be suitable candidates for antidiabetic agents via antioxidant effects (23).

The probable mechanism underlying garlic's hypoglycemic effects is increasing either the pancreatic secretion of insulin from the beta cells and its release from bound insulin or insulin sensitivity $(14,16)$. Also, garlic has been reported to spare insulin from sulfhydryl group (which is known to inactivate insulin) by some compounds identified in garlic such as allicin; which can effectively combine with cysteine residues and enhance insulin sensitivity $(10,16)$.

Although how exactly garlic improves SOD, GPX, and CAT activities is still unclear, several possible mechanisms could be put forward to explain this effect.

The antioxidant activity of Allium sativum could be attributed mainly to a variety of sulphur-containing compounds and their precursors (21). The primary sulfur-containing compound of intact garlic bulb is $\gamma$-glutamyl cysteine which can be hydrolyzed and oxidized to form alliin (2). Alliin is converted to odoriferous thiosulfinate allicin by alliinase after processing such as crushing, cutting, chewing or dehydration (21). Scientific evidence shows that allicin, diallyl disulphide and diallyl trisulphide appeared to be the main antioxidative compounds (15). Allicin was also found to reduce level of blood glucose in male Wistar rats with diabetes induced by streptozotocin (18).

Furthermore, beneficial effects of garlic may be attributed to the induction of an antioxidant enzymes such as of glutamate-cysteine ligase (GCL) that protects cells from oxidative injury by increasing glutathione (GSH) content (6). Expression of genes encoding for GCL in turn is regulated by nuclear factor erythroid-2-related factor 2 (Nrf2). Ajoene (a chemically stable garlic by-product), activates protein kinase C-delta (PKCdelta) that promotes the Nrf2-dependent antioxidant defense system and induce GCL (6).

The other probable protective mechanism(s) of garlic against oxidative damage may be linked to its bioactive components which perhaps chelate the metal ions $(\mathrm{Cu}, \mathrm{Zn}, \mathrm{Mn})$ thereby scavenge the superoxide ions and subsequently inhibiting the oxidation of protein moieties and thus contributing to the healthy cellular redox status (24).

Also, in the assessment of the antioxidant capacities of garlic extracts, the ethyl acetate extract showed the highest ferric reducing and radical scavenging activities (1). In addition, the antioxidant activity can be related to other bioactive compounds such as dietary fibers, microelements (especially Se) and polyphenols (17).

In the present study, garlic administration also increased oxidative defence system significantly in control rats, showing that administration of garlic homogenate in controls activates nearly the same mechanisms and pathways that augment the antioxidant defense system.

The underlying mechanisms responsible for the increased injury in diabetic myocardium are not fully understood. However, excessive free radical generation and/or depleted endogenous antioxidant defense system have been implicated (27).

The key histopathological finding was that myocardium of diabetic animals showed myonecrosis with edema, inflammatory cells and separation of cardiac muscle fibers. Treatment of rats with garlic homogenate improved heart tissue damages shown as mild edema and inflammatory cells. 
In conclusion, garlic inhibits lipid peroxidation and activates free radical scavengers in both blood and heart of the streptozotocin treated animals. Therefore, garlic supplementation may help in the protection against the free radicals production in diabetes.

\section{Acknowledgement}

This research was supported by a grant from the Applied Drug Research Center, Tabriz University of Medical Sciences, Tabriz, Iran.

\section{REFERENCES}

1. Abrahim NN, Kanthimathi MS, Abdul-Aziz A: Piper betle shows antioxidant activities, inhibits MCF-7 cell proliferation and increases activities of catalase and superoxide dismutase. BMC. Complement Altern. Med. 15, 12-220 (2012)

2. Amagase H, Petesch BL, Matsuura H, Kasuga S, Itakura Y: Intake of garlic and its bioactive components. J. Nutr. 131, 955S-962S (2001)

3. Badalzadeh R, Layeghzadeh N, Alihemmati A, Mohammadi M: Beneficial effect of troxerutin on diabetes induced vascular damages in rat aorta: histopathological alterations and antioxidation mechanism. Int. J. Endocrinol. Metab. 13, e25969 (2015)

4. Bao H, Chen L: [Icariin reduces mitochondrial oxidative stress injury in diabetic rat hearts]. Zhongguo. Zhong. Yao. Za. Zhi. 36, 1503-1507 (2011)

5. Baynes JW: Role of oxidative stress in development of complications in diabetes. Diabetes 40, 405-412 (1991)

6. Capasso A: Antioxidant action and therapeutic efficacy of Allium sativum L. Molecules 18, 690-700 (2013)

7. Chan JY, Yuen AC, Chan RY, Chan SW: A review of the cardiovascular benefits and antioxidant properties of allicin. Phytother. Res. 27, 637-646 (2013)

8. Cheng AYY, Josse RG: Intestinal absorption inhibitors for type 2 diabetes mellitus: prevention and treatment. Drug Discov. Today Ther. Strateg. 1, 201-206 ( 2004)

9. Drobiova H, Thomson M, Al-Qattan K, Peltonen-Shalaby R, Al-Amin Z, Ali M: Garlic increases antioxidant levels in diabetic and hypertensive rats determined by a modified peroxidase method. Evid. Based Complement Alternat. Med. 2011, ID 703049 (2011)

10. El-Demerdash FM, Yousef MI, El-Naga NI: Biochemical study on the hypoglycemic effects of onion and garlic in alloxan-induced diabetic rats. Food Chem. Toxicol. 43, 57-63 (2005)

11. Erejuwa OO: Management of diabetes mellitus: could simultaneous targeting of hyperglycemia and oxidative stress be a better panacea? Int. J. Mol. Sci. 13, 2965-2972 (2012)

12. Fiorentino TV, Prioletta A, Zuo P, Folli F: Hyperglycemia-induced oxidative stress and its role in diabetes mellitus related cardiovascular diseases. Curr. Pharm. Des. 19, 5695-5703 (2013)

13. Giugliano D, Ceriello A, Paolisso G: Oxidative stress and diabetic vascular complications. Diabetes Care 19, 257-267 (1996)

14. Jain RC, Vyas CR: Garlic in alloxan-induced diabetic rabbits. Am. J. Clin. Nutr. 28, 684-685 (1975)

15. Kim SM, Kubota K, Kobayashi A: Antioxidative activity of sulfur-containing flavor compounds in garlic. Biosci. Biotech. Bioch. 61, 1482-1485 (1997)

16. Kumar R, Chhatwal S, Arora S, Sharma S, Singh J, Singh N, Bhandari V, Khurana A: Antihyperglycemic, antihyperlipidemic, anti inflammatory and adenosine deaminase low ring effects of garlic in patients with type 2 diabetes mellitus with obesity. Diabetes Metab. Syndr. Obes. 6, 49-56 (2013)

17. Lanzotti V: The analysis of onion and garlic. J. Chromatogr. A. 1112, 3-22 (2006)

18. Liu Y, Qi H, Wang Y, Wu M, Cao Y, Huang W, Li L, Ji Z, Sun H: Allicin protects against myocardial apoptosis and fibrosis in streptozotocin-induced diabetic rats. Phytomedicine 19, 693-698 (2012)

19. Mohammadi M, Ghaznavi R, Keyhanmanesh R, Sadeghipour HR, Naderi R, Mohammadi H: Voluntary exercise prevents lead-induced elevation of oxidative stress and inflammation markers in male rat blood. Sci. World J. 2013, ID 320704 (2013)

20. Negre-Salvayre A, Salvayre R, Augé N, Pamplona R, Portero-Otín M: Hyperglycemia and glycation in diabetic complications. Antioxid. Redox Signal. 11, 3071-3109 (2009) 
21. Nishimura H, Higuchi O, Tateshita K: Antioxidative activity of sulfur-containing compounds in Allium species for human LDL oxidation in vitro. Biofactors 21, 277-280 (2004)

22. Park SA, Choi MS, Jung UJ, Kim MJ, Kim DJ, Park HM, Park YB, Lee MK: Eucommia ulmoides Oliver leaf extract increases endogenous antioxidant activity in type 2 diabetic mice. J. Med. Food 9, 474-479 (2006)

23. Queiroz YS, Ishimoto EY, Bastos DH, Sampaio GR, Torres EA: Garlic (Allium sativum L.) and ready-to-eat garlic products: In vitro antioxidant activity. Food Chem. 115, 371-374 (2009)

24. Rajani Kanth V, Uma Maheswara Reddy P, Raju TN: Attenuation of streptozotocin-induced oxidative stress in hepatic and intestinal tissues of Wistar rat by methanolic-garlic extract. Acta Diabetol. 45, 243-251 (2008)

25. Somi MH, Hajipour B, Asl NA, Estakhri R, Azar AN, Zade MN, Haghjou AG, Vatankhah AM: Pioglitazone attenuates ischemia/reperfusion-induced liver injury in rats. Transplant. Proc. 41, 4105-4109 (2009)

26. Wang GG, $\mathrm{Li} \mathrm{W}, \mathrm{Lu} \mathrm{XH}, \mathrm{Zhao} \mathrm{X}, \mathrm{Xu} \mathrm{L}$ : Taurine attenuates oxidative stress and alleviates cardiac failure in type I diabetic rats. Croat. Med. J. 54, 171-179 (2013)

27. Youn JY, Park HY, Cho KH: Anti-hyperglycemic activity of Commelina communis L.: inhibition of alphaglucosidase. Diabetes Res. Clin. Pract. 66, 149-155 (2004) 